\title{
Ethnomedicinal knowledge among the Malayali tribal of Chitteri hills, Eastern Ghats, Tamil Nadu, India
}

\author{
R. Prabakaran', T. Senthil Kumar* \\ 'Department of Botany, Ramakrishna Mission Vivekananda College (Autonomous), Mylapore, Chennai-600004, \\ Tamil Nadu, India, ${ }^{2}$ Department of Botany, Bharathidasan University, Tiruchirappalli-620 024, Tamil Nadu, India
}

Received: December 14, 2020 Revised: February 24, 2021 Accepted: March 01, 2021 Published: March 19, 2021

\section{*Corresponding Author:} T. Senthil Kumar* E-mail: senthilbdc@bdu.ac.in

\section{ABSTRACT}

The present study was aimed to document the ethnomedicinal knowledge among the Malayali tribal of Chitteri hills Eastern Ghats of Tamil Nadu, India. Field visits were made to the Chitteri hills every month covering all seasons. Interviews with traditional healers and other knowledgeable inhabitants and farmers were conducted. The Malayali tribal people of Chitteri hills use 320 plant species for their day-to-day life, this ethnobotanical exploration revealed they were the habit of using around 216 species of medicinal plants belonging to 200 genera under 45 families. Malayali tribes use morphological characters such as bark surface, leaf colour, leaf taste and exudates, underground plant parts and ecology of species as criteria for identification of 135 species belongs to 105 genera under 46 families. The documentation of the knowledge of Malayali tribal identification of plants of Chitteri hills is to be accorded top priority in the preservation of our ancient traditional knowledge.

KEYWORDS: Ethnobotany, Chitteri, Tamil Nadu, Eastern Ghats and Malayali

\section{INTRODUCTION}

Traditional knowledge of taxonomy is developed from a basic human tendency to recognize plants that are imposed by nature. It is developed from the unique history and culturally defined beliefs, behaviors and preferences of particular traditional societies rooted in a clearly defined geographical area and transmit their knowledge to their offspring's. The universal identification of plants had been ubiquitous since the evolution of systematic botany. Evolution of taxonomy triggered botanists, to explore variety of plant species universally on their biological properties and evolved into the present modern ethnobotany, which emphasize on their, growth pattern and chemical compositions in traditional communities need. With the passage of time, they have developed a great deal of knowledge on the use of plants and plant products. The tribal have their own scientific knowledge of technology and they are still considered to be primitive and traditional bounded. The knowledge is very dynamic and is strongly influenced by indigenous creativity, innovation, rooted in geographical and cultural cognition. The knowledge is very vulnerable to degradation and even complete loss. In this perspective a rich diversity of flora of Chitteri hills was chosen for the study to document with objectives to reveal the criteria used by the Malayali of Chitteri hills use morphological characters and ecology of species as criteria for identification.

\section{MATERIALS \& METHODS}

\section{Study Area}

The present study area, Chitteri hills, a part of Southern Eastern Ghats, is situated in Pappireddipatti revenue taluk of Dharmapuri district in Tamil Nadu, India. Dharmapuri district has the second highest forest cover in relation to the total geographical area, satisfying the criterion of optimum forest cover of $23.62 \%$ in its geographical area. The district accounts for $14.3 \%$ of the total forest area of the Tamil Nadu.

Chitteri is situated towards North East of Salem district within the geographical limit of $78^{\circ} 15^{\prime}-78^{\circ} 45^{\prime} \mathrm{E}$, longitude and $11^{\circ} 44^{\prime}$ $12^{\circ} 08^{\prime} \mathrm{N}$, latitude (Figure-1) and occupies an area of about $654.22 \mathrm{Km}^{2}$. Chitteri hills form a compact block consisting of several hill ranges and contain tangled ridges and ravines running in the Northeast and Southwest directions, enclosing many narrow valleys, rivers such as Kallar, Varattar, Kambalai, Anaimaduvu, Kovilar, Sholaiyar and Pungamadauvu rivers and their tributaries drain the area. These rivers are ephemeral in

Copyright: $\odot$ The authors. This article is open access and licensed under the terms of the Creative Commons Attribution License (http:// creativecommons.org/licenses/by/4.o/) which permits unrestricted, use, distribution and reproduction in any medium, or format for any purpose, even commercially provided the work is properly cited. Attribution - You must give appropriate credit, provide a link to the license, and indicate if changes were made. 
nature and structurally controlled in their flow. The mean maximum and minimum annual temperatures of the study area are $39.5^{\circ} \mathrm{C}$ and $19^{\circ} \mathrm{C}$, and $31^{\circ} \mathrm{C}$ and $18^{\circ} \mathrm{C}$ respectively in winter, average rainfall ranges from $800-1000 \mathrm{~mm}$ (Harur Forest Office Report, 2007).The Malayali tribes are the most and dominant significant tribes in Chitteri hills of Tamil Nadu. Malayali are the largest Scheduled Tribe constituting 47\% of the state scheduled tribal population with a population of 11,482 (Census, 2011). There are 60 villages, out of these, 6 villages are located in plains and 54 villages are located in hill tops.

\section{Field Visits}

Field visits were made to the Chitteri hills every month covering all seasons during the period October 2009 - March 2013. Interview and data gathering methods were followed by (Schultes, 1962; Jain, 1995; Rao \& Hajra, 1987) Interviews with traditional healers and other knowledgeable inhabitants and farmers were conducted in order to understand how Malayali tribes identify and utilize plants. In addition, we consulted the who is familiar with the study area. Voucher specimens were collected for the purpose of identification and deposited at Vivekanandha College of Arts and Sciences for Women herbarium.

\section{Documentation of Ethnoidentification of Plants Knowledge}

The respondents or informants have been selected for the study based on the following criteria, prevalence of ethnobotanical knowledge in villages and willingness of respondents to share the knowledge. All the respondents are men belong to 25 to $85 \mathrm{yrs}$ of age group. Most of the respondents are illiterate or even never crossed primary education.

Data were collected from the tribes using two different methods: 1) The knowledgeable informants are taken to the field and collection of plants specimens with uses of the plants and 2) The other way is to collect all plants available in the village, show them to these informants one by one, and record the information about them. The data were gathered in a series of questionnaire, structured, semi-structured and unstructured interviews regarding plant uses, identification during several field trips. Random interviews with a different sub sect of tribal were used to verify data already collected regarding indigenous knowledge for identification of plant species of Malayali tribes of Chitteri hills was documented.

\section{RESULTS AND DISCUSSION}

Periodical trips were made to the study area covering all the hamlets in the hills. Such frequent visits to tribal hamlets helped us to establish a good rapport with the natives, which aided us in the collection day today life. Much patience was needed for gathering information from tribes. Only after successive visits the native divulge their knowledge of plants, especially used for medicine and identification. A cordial relationship was established with them and they feel quite at ease in our company.
Most of the tribes are illiterate and this rules out the possibility of using questionnaires as means of collecting data, though we prepared questionnaires for ethno medicinal plants and traditional knowledge. Dialogues, conversations and subsequent recording of data are made. The authenticity of the information gathered is verified subsequent field trips to other areas with other persons. Such trips helped in verifying the validity of the other related uses. From such field visits, we recorded that Malayali tribal people of Chitteri hills are in the habit of using 320 species for their day today life.

\section{Enumeration of Ethnobotanical Plants}

The present ethnobotanical exploration revealed that the Malayali tribal people of Chitteri hills are in the habit of using around 216 species of medicinal plants belonging to 200 genera under 45 families. The families were named as per APG-III classification 2009.

The Malayali tribes of Chitteri hills prefer to use species from their native forest and species from surrounding areas. Among the plants used by them Apocynaceae topping the list with 37 species, Fabaceae (29 species), Acanthaceae and Rutaceae each listed with 16 species. Based on the habit, ethno medicinal plants of Chitteri hills fall under various categories such as trees, shrubs, herbs, climbers and lianas. Among them, trees and herbaceous growth forms dominate over other growth forms.

\section{Identification of Plants by Tribal}

\section{Morphological characters}

The Malayali tribes use morphological characters and ecology of species as criteria for identification of 135 species (Voucher specimen number from $\mathrm{Vi}-432$ to $\mathrm{Vi}-567)$ belongs to 105 genera under 46 families. Morphological characters are often used to recognize plants of which vegetative features are more commonly used than floral features. Malayali tribes also identify plants based on morphological characters with other associated characters such as taste, colour, succulence of leaves and exudates.

\section{Bark characters}

The term bark denotes the tissues outside the vascular cambium of the axis, in either a primary or secondary state of growth. In botanical sense bark is that layer of tissue accumulated on the surface of the plant axis as a result of the activity of the phellogen. Bark is an outwardly visible and prominent macro character especially in trees. Malayali use bark characters as the main criterion for identifying tree species with other characters such as nature and colour of leaf and presence or absence of glands as supportive characters.

Bark features such as nature of the surface, thickness of bark and exudates are used by the Malayali as criteria for identification. Among the species, studied Malayali tribal classified 97 species based on bark features without ambiguity (Table 1). 
Table 1: Identification of plants with bark characters in Chitteri hills by Malayali tribes

\begin{tabular}{|c|c|c|c|c|}
\hline S. No. & Botanical Name & Family & Local name & Bark Characters \\
\hline 1. & Acacia leucophloea (Roxb.) Willd. & Fabaceae & Velvelam/Velamaram & Smooth Bark \\
\hline 2. & Alangium salvifolium (L.f.) Wang. & Alangiaceae & Azhingi & Rough Bark \\
\hline 3. & Albizia amara (Roxb.) Bolvin & Fabaceae & Unzai & Flaked Bark \\
\hline 4. & Albizia chinensis (Osbeck.) Merr. & Fabaceae & Selavengi & Rough Bark \\
\hline 5. & Albizia lebbeck (L.) Willd. & Fabaceae & Pattaisilai/Vagai & Rough Bark \\
\hline 6. & Albizia procera L. & Fabaceae & Kudumaduramaram & Smooth\&Thick Bark \\
\hline 7. & Anacardiu moccidentale L. & Anacardiaceae & Mundhiri & Rough Bark \\
\hline 8. & Anogeissus latifolia (Roxb. ex DC.) Wall. ex Guill. \&Perr. & Combretaceae & Namaimaram & Flaked \& Thick Bark \\
\hline 9. & Artocarpus heterophyllus Lam. & Moraceae & Palamaram & Rough Bark \\
\hline 10. & Atalantia monophylla (L.) DC. & Rutaceae & KaattuElumichai & Prickled Bark \\
\hline 11. & Bauhinia tomentosa L. & Fabaceae & Aachamaram/Pathinimaram & Rough Bark \\
\hline 12. & Bombax ceiba L. & Bombacaceae & Ilavu & Prickled Bark \\
\hline 13. & Bridelia crenulataRoxb.Buchanania axillaris (Desr.) T.P. & Euphorbiaceae & Marivaengai & Rough Bark \\
\hline 14. & Ramamoorthyinc.J.Saldanha\& Nicolson & Anacardiaceae & Sulluki/Saraparuppumaram & Rough and Segmented bark \\
\hline 15. & Canthium dicoccum (Gaertn.) Teijsm. \&Binn. & Rubiaceae & Nekkini & Rough Bark \\
\hline 16. & Capparis zeylanica L. & Capparaceae & Athandai & Prickled Bark \\
\hline 17. & Cassia fistula L. & Fabaceae & Konnai/Sarakonnai & Smooth \& Thick Bark \\
\hline 18. & Cassia siamea Lam. & Fabaceae & Thagaraimaram & Smooth Bark \\
\hline 19. & Ceiba pentandra (L.) Gaertn. & Bombacaceae & Ilavu & Prickled Bark \\
\hline 20. & Chloroxylon swietenia DC. & Rutaceae & Purasamaram & Rough Bark \\
\hline 21. & Chukrasia tabularis A. Juss. & Meliaceae & Magombumaram & Rough Bark \\
\hline 22. & Cleistanthus collinus (Roxb.) Hook.f. & Euphorbiaceae & Oduvanthazhai & Rough Bark \\
\hline 23. & Commiphora caudata (Wight. \&Arn.) Engl. & Buseraceae & Pachakiluvai & Smooth\& Flaked Bark \\
\hline 24. & Cordia obliquaWilld. & Boraginaceae & Vallukumaram & Rough Bark \\
\hline 25. & Cordia wallichii G. Don. & Boraginaceae & Panthekku & Rough Bark \\
\hline 26. & Dalbergia lanceolariaL.f. & Fabaceae & Eetimaram & Rough Bark \\
\hline 27. & Dalbergia latifoliaRoxb. & Fabaceae & Eetimaram & Rough \&Thick Bark \\
\hline 28. & Diospyros ebenum J. Koen. ex Retz & Ebenaceae & Karungali & Roughand Segmented bark \\
\hline 29. & Diospyros ferrea (Willd.) Bakh. & Ebenaceae & Irumbuli & Rough Bark \\
\hline 30. & Diospyros melanoxylonRoxb. & Ebenaceae & Thumbaranmaram & Rough and Segmented bark \\
\hline 31. & Diospyros montanaRoxb. & Ebenaceae & Vellungumaram & Prickled Bark \\
\hline 32. & Diospyros ovalifolia Wight. & Ebenaceae & Kari maram & Rough Bark \\
\hline 33. & Erythroxylum monogynumRoxb. & Erythroxylaceae & Sembulichaan/Devadhaaru & Flaked Bark \\
\hline 34. & Eucalyptus tereticornis Smith & Myrtaceae & Thailamaram & Flaked Bark \\
\hline 35. & Ficus racemosa L. & Moraceae & Athimaram & Thick Bark \\
\hline 36. & Ficus benghalensis L. & Moraceae & Aalamaram & Smooth Bark \\
\hline 37. & Ficus microcarpaL.f. & Moraceae & Kalichi & Smooth Bark \\
\hline 38. & Ficus racemosa L. & Moraceae & Atthi & Smooth Bark \\
\hline 39. & Ficus religiosa L. & Moraceae & Arasamaram & Smooth Bark \\
\hline 40. & Ficus virensAit. & Moraceae & Irali/Maraichi & Smooth Bark \\
\hline 41. & Filicium decipiens (Wight. \&Arn.) Thwaites & Sapindaceae & Jannimaram & Smooth Bark \\
\hline 42. & Gardenia gummiferaL.f. & Rubiaceae & Kambimaram & Thick Bark \\
\hline 43. & Glycyrrhiza glabra L. & Fabaceae & Athimadhuram & Smooth Bark \\
\hline 44. & Gmelina arboreaRoxb. & Lamiaceae & Kumizhamaram & Rough Bark \\
\hline 45. & Grevillea robusta A. Cunn. ex R. Br. & Proteaceae & Silver rook & Rough Bark \\
\hline 46. & Gyrocarpus americanusJacq. & Hernandiaceae & Thanku & Smooth Bark \\
\hline 47. & Ixora pavettaAndr. & Rubiaceae & Koraamaram & Rough Bark \\
\hline 48. & Lannea coromandelica (Houtt.) Merr. & Anacardiaceae & Kulimathi/Odhiyamaram & Smooth\&Thick Bark \\
\hline 49. & Ligustrum perrottetiiA.DC.ex DC. & Oleaceae & Pasarmaram & Smooth Bark \\
\hline 50. & Limonia acidissima L. & Rutaceae & Vila & Prickled Bark \\
\hline 51. & Madhuca longifolia (L.) Machr. & Sapotaceae & Kaatuilluppai & Rough Bark \\
\hline 52. & Mallotus philippensis (Lam.) Muell. Arg. & Euphorbiaceae & Thiruchilaimaram & Rough Bark \\
\hline 53. & Mangifera indica L. & Anacardiaceae & Maamaram & Rough Bark \\
\hline 54. & Memecylon eduleRoxb. & Melastomataceae & Allan maram & Rough and Segmented bark \\
\hline 55. & Mimusop selengi $\mathrm{L}$. & Sapotaceae & Molluva/Magizhamaram & Rough Bark \\
\hline 56. & Mitragyna parvifolia (Roxb.) Korth. & Rubiaceae & Neerkadampai & Flaked Bark \\
\hline 57. & Morinda coreiaBuch. Ham. & Rubiaceae & Nunamaram & Roughand Segmented bark \\
\hline 58. & Naringi crenulata (Roxb.) Nicolson & Rutaceae & Naivila/Porivilangamaram & Rough Bark \\
\hline 59. & Nothopegia colebrookeana (Wight.) Blume & Anacardiaceae & Kattumathi/Kattuma & Rough \&Thick Bark \\
\hline 60. & Phyllanthu semblica L. & Phyllanthaceae & Periyanelli & Smooth Bark \\
\hline 61. & Pithecellobium dulce (Roxb) Benth. & Fabaceae & Konakai/Kodukkaaipuli & Rough Bark \\
\hline 62. & Pittosporumn apaulense (DC.) Rehder\&E.H.Wilson & Pittosporaceae & Vellaimathi & Smooth Bark \\
\hline 63. & Pleurostylia opposita (Wall.) Alston & Celastraceae & Sutholingi & Rough Bark \\
\hline 64. & Plumeria rubra L. & Apocynaceae & Arali & Smooth Bark \\
\hline 65. & Polyalthia cerasoides (Roxb.) Bedd. & Annonaceae & Senthalamaram & Rough and Segmented bark \\
\hline
\end{tabular}




\begin{tabular}{|c|c|c|c|c|}
\hline S. No. & Botanical Name & Family & Local name & Bark Characters \\
\hline 66. & Pongamia pinnata (L.) Pierre. & Fabaceae & Pungamaram & Rough Bark \\
\hline 67. & Premna tomentosa Willd. & Lamiaceae & Ponnari & Rough Bark \\
\hline 68. & Pterocarpus marsupiumRoxb. & Fabaceae & Vengai & Rough \& Thick Bark \\
\hline 69. & Santalum album L. & Santalaceae & Santhanam & Rough Bark \\
\hline 70. & Schleichera oleosa (Lour.) Oken. & Sapindaceae & Sakattamaram & Rough Bark \\
\hline 71. & Semecarpus anacardium L. & Anacardiaceae & Serra maram & Rough \& Thick Bark \\
\hline 72. & Shorea roxburghii G. Don. & Dipterocarpaceae & Silari/Kungiliyam & Rough \&Thick Bark \\
\hline 73. & Strychnos nux-vomica L. & Loganiaceae & Yetti & Rough Bark \\
\hline 74. & Strychno spotatorum L. & Loganiaceae & Thethamaram & Rough Bark \\
\hline 75. & Swietenia mahagoni (L.) Jacq. & Meliaceae & Mahagony & Rough Bark \\
\hline 76. & Syzgium cumini (L.) Skeels & Myrtaceae & Naval & Flaked Bark \\
\hline 77. & Tamarindus indica L. & Fabaceae & Puliyamaram & Rough Bark \\
\hline 78. & Tectona grandis L.f. & Lamiaceae & Thekku & Rough Bark \\
\hline 79. & Terminalia arjuna (DC.) Wight. \&Arn. & Combretaceae & Neermathi & Smooth, Thick \& Flaked Bark \\
\hline 80. & Terminalia bellirica (Gaertn.) Roxb. & Combretaceae & Thandri & Rough \& Thick Bark \\
\hline 81. & Terminaliac hebulaRetz. & Combretaceae & Kadukai & Rough Bark \\
\hline 82. & Terminalia crenulata Roth. & Combretaceae & Karumarudhu & Rough and Segmented bark \\
\hline 83. & Terminalia tomentosa W. \& A. & Combretaceae & Pillaimarudu & Rough \& Thick Bark \\
\hline 84. & Thespesia populnea (L.) Soland. ex Corrêa & Malvaceae & Poovarasu & Rough Bark \\
\hline 85. & Thevetia peruviana K. Schum. & Apocynaceae & Thangaarali & Rough Bark \\
\hline 86. & Vitex altissimaL.f. & Verbenaceae & Mayiladi & Smooth Bark \\
\hline 87. & Vitex negundo L. & Verbenaceae & Vellainochi & Smooth Bark \\
\hline 88. & Wrightia tinctoria (Roxb.) R. Br. & Apocynaceae & Vetpaalai & Smooth Bark \\
\hline
\end{tabular}

Rough bark, rough segmented bark, smooth bark, prickled bark, fissured bark and flaked bark are the six different types of bark surfaces recognized by the Malayali in the tree species of Chitteri hills. The trees recorded in the study possess Rough bark and belongs to 50 species, 42 genera under 38 families. The tree possess Smooth bark belongs to 26 species, 23 genera under 17 families. The rough and segmented bark reported in 7 tree species belong to 5 genera under 5 families. The Prickled bark is reported in 6 tree species. The Flaked bark reported in 8 species belongs to 8 genera. Malayali identify 19 trees that possess thick bark and. They also identify 2 species by inner colour of the bark, they are Bridelia crenulata Roxb. and Pleurostylia opposita (Wall.) Alston, possess red and white colour inner bark respectively.

Bark has been used as a means of recognition of trees by many tribal societies across the world. For example, the tribes in West Africa classified Adansonia digitata L. the Baobab tree into the following four types using bark character such as colour and surface of bark: 1.) Smooth pink bark, 2.) Rough grey bark, 3.) Smooth grey bark and 4.) Black bark (Assogbadjo et al., 2006). Batoros and Bakigas in Western Uganda recognize trees based on life forms. Identifying trees by their architecture is no problem for them (Kakudidi, 2004).

Modern day field-botanists tend to use vegetative features such as bark characters, leaf characters, overall branching pattern and life form for on-the-spot identification of trees. The above mentioned a few publications in which this approach has been standardized.

\section{Plant Exudates}

The secretory spaces in the form of cavities or canals are formed by schizogeny or by lysigeny or sometimes by both phenomena combined. Laticifers are cells or series of fused cells containing fluid called latex and forming systems that permeate various tissues of the plant body.

Any discharge from the plants named as exudates by Malayali tribes of Chitteri hills. They use colour of exudates, change of colour in the exudates and the drying characters of the exudates as important criteria for identifying plant species. Malayali tribes consider the milky and white latex as pal in Tamil meaning milky secretion. They recognize 25 milky and white latex yielding plants belonging to 21 genera and 7 families (Table-2). The diversity of habit of these species is trees (14 species), shrubs (3 species), straggler (3 species), climber ( 3 species) and herb (2 species).Seven species are reported with colourless latex yielding plants, 5 species are reported with red exudates yielding plants. Eight species reported as gum yielding plants and 5 species are resin yielding plants.

For example, the bark of Pterocarpus marsupium naturally discharges red coloured latex in a steady continuous flow. Initially it is non viscous and later on it becomes viscous. After a period of $48 \mathrm{hrs}$, the red coloured latex changes into a semi solid black substance. The bark of Buchanania axillaris discharges watery latex very slowly without any cut open, after 2-3 hrs it turns into semi solid. Without any cut open bark steadily discharge of viscous milky latex is characteristic of Ficus benghalensis, F. glomerata, F. microcarpa and Freligiosa. The watery latex from the bark of Cassine glauca is natural one and turns into semisolid colourless gum in a few hours after discharge. The bark of Semecarpus anacardium is cut open, it discharges red coloured exudates very slowly and it turns into a semi solid black mass. On physical contact with the latex is injurious to the skin causing blisters.

The keen observation of the natural phenomena the Malayali tribes of Chitteri hills have is quite surprising. To cite an example, 
Table 2: Identification of plants with leaves, exudates and ecological characters in Chitteri hills by Malayali tribes

\begin{tabular}{|c|c|c|c|c|}
\hline S. No. & Botanical Name & Family & Local Name & Identification Characters \\
\hline 1. & Agave angustifolia L. & Agavaceae & Katarali & Succulence leaves \\
\hline 2. & Albizia procera L. & Fabaceae & Kudumadurai & Riparian \& Watery latex \\
\hline 3. & Aloe vera (L.) Burm.f. & Liliaceae & Katralai & Succulence leaves\& Watery latex \\
\hline 4. & Andrograhis alata (Vahl.) Nees. & Acanthaceae & Siriyanangai & Taste \\
\hline 5. & Andrograhis paniculata (Burm.f.) Wallich ex Nees. & Acanthaceae & Nilavembu & Taste \\
\hline 6. & $\begin{array}{l}\text { Anogeissus latifolia (Roxb. ex DC.) Wall. exGuill. } \\
\text { \&Perr. }\end{array}$ & Combretaceae & Namaimaram/Vetkaali gum & Colour young leaves \& Gum \\
\hline 7. & Aristolochia indica L. & Aristolochiaceae & Aduthinapalai & Colour leaves \\
\hline 8. & Artocarpus heterophyllus Lam. & Moraceae & Kaatupala & Milky latex \\
\hline 9. & Artocarpus hirsutusL.f. & Moraceae & Kari palamaram & Milky latex \\
\hline 10. & Asparagus recemosaWilld. & Liliaceae & Thaineervetankizangu & Tuber \\
\hline 11. & Bombax ceiba L. & Bombacaceae & Ilavu & Riparian \\
\hline 12. & Bridelia crenulataRoxb. & Euphorbiaceae & Marivaengai & Red latex \\
\hline 13. & $\begin{array}{l}\text { Buchanania axillaris (Desr.) T.P. Ramamoorthyinc. J. } \\
\text { Saldanha\& Nicolson }\end{array}$ & Anacardiaceae & Sulluki/Saraparuppumaram & Watery latex \\
\hline 14. & Calatropis procera Br. & Apocynaceae & Vellaerukkan & Milky latex \\
\hline 15. & Calotropis gigantea (L.) R.Br. & Apocynaceae & Erukkan & Milky latex \\
\hline 16. & Carulluma adscendensvar. attenuata Wight & Apocynaceae & Kallumullian & Watery latex \\
\hline 17. & Cassine glauca (Rottb.) Kuntze & Celastraceae & Eelimaram & Gum \\
\hline 18. & Catunaregum spinosa (Retz.) Poiret & Rubiaceae & Marakarai & Root \\
\hline 19. & Chloroxylon swietenia DC. & Rutaceae & Purasamaram & Watery latex \\
\hline 20. & Clerodendrum inerme (L.) Gaerrtner & Verbenaceae & Nar SanguIllai & Taste of leaves \\
\hline 21. & Cordia wallichii G. Don. & Boraginaceae & Panthekku & Watery latex \\
\hline 22. & Cosmostigma racemosum (Roxb.) Wight & Apocynaceae & Padameratti & Milky latex \\
\hline 23. & Crateva magna DC. & Capparaceae & - & Tuber \\
\hline 24. & Croton bonplandianus Ballion & Euphorbiaceae & Poondu & Watery latex \\
\hline 25. & Cryptolepis grandiflora Wight. & Apocynaceae & Attankodi/Matangodi & Milky latex \\
\hline 26. & Curculigo orchioidesGaertner & Hypoxidaceae & Nilapanaikilangu & Tuber \\
\hline 27. & Decalepis hamiltoni Wight. \&Arn. & Apocynaceae & Mavilangum & Tuber \& Milky latex \\
\hline 28. & Dioscorea pentaphylla L. & Dioscoreaceae & Vallikilangu & Tuber \\
\hline 29. & Dioscorea bulbifera L. & Dioscoreaceae & Kavalaikizangu & Tuber \\
\hline 30. & Dioscorea oppositifolia L. & Dioscoreaceae & Malaiyankilangu & Tuber \\
\hline 31. & Diospyrosferrea (Willd.) Bakh. & Ebenaceae & Irumbuli & Gum \\
\hline 32. & Drynaria quercifolia (L.) J. Sm. & Polypodiaceae & Attukaalkilangu & Tuber \\
\hline 33. & Eucalyptus tereticornis Smith & Myrtaceae & Thailamaram & Gum \\
\hline 34. & Euphorbia antiaquorumL. & Euphorbiaceae & Sathurakalli & Milky latex \\
\hline 35. & Euphorbia herterophylla L. & Euphorbiaceae & Venmaikolunthu & Milky latex \\
\hline 36. & Ficus benghalensis L. & Moraceae & Alamaram & Milky latex \\
\hline 37. & Ficus glomerata Roxb. & Moraceae & Athimaram & Milky latex \\
\hline 38. & Ficus infectoria Willd. & Moraceae & Malaiitchi & Milky latex \&Riparian \\
\hline 39. & Ficus microcarpa L. f. & Moraceae & Kalarasan & Milky latex \\
\hline 40. & Ficus racemosa L. & Moraceae & Atthi & Milky latex \& Riparian \\
\hline 41. & Ficus religiosa $\mathrm{L}$. & Moraceae & Arsamaram & Milky latex \\
\hline 42. & Flueggela virosa (Willd.) Baillon & Euphorbiaceae & - & Colourleaves \\
\hline 43. & Gardenia gummifera L.f. & Rubiaceae & Kambimaram & Resin \\
\hline 44. & Gardenia resinifera Roth. & Rubiaceae & Kambimaram & Resin \\
\hline 45. & Grevillea robusta A. Cunn. ex R. Br. & Proteaceae & Malaisavuku & Resin \\
\hline 46. & Gymnema sylvestre (Retz.) R.Br.ex Roemer \&Schultes & Apocynaceae & Sirukurinjan & Milky latex \&Taste of leaf \\
\hline 47. & Hardwickia binataRoxb. & Fabaceae & Achamaram & Resin \\
\hline 48. & Hemidesmus indicus (L.) R.Br. & Apocynaceae & Sirumolikizhangu & Tuber \& Milky latex \\
\hline 49. & Hiptage benghalensis (L.) Kurz. & Malpighiaceae & Suthalakodi & Leaf Glands \\
\hline 50. & Icnocarpus rutescens (L.) R.Br. & Apocynaceae & Palvallikodi & Milky latex \\
\hline 51. & Limonia acidissima L. & Rutaceae & Vila & Gum \\
\hline 52. & Litsea oleoides (Meissner) Hook. f. & Lauraceae & - & Colour leaves \\
\hline 53. & Madhuca longifolia (L.) Machr. & Sapotaceae & Kaatuilluppai & Milky latex, Colour leaves\&Riparian \\
\hline 54. & Maerua ablongifolia (Forsskal) A.Rich. & Capparaceae & Pumisarkaraikizhangu & Tuber \\
\hline 55. & Mallotus philippensis (Lam.) Muell. Arg. & Euphorbiaceae & Thiruchilaimaram & Leaf Glands \\
\hline 56. & Mangifera indica L. & Anacardiaceae & $\mathrm{Ma}$ & Riparian \\
\hline 57. & Manilkara hexandraDubard & Sapotaceae & - & Riparian \\
\hline 58. & Marsdenia tenacissima (Roxb.) Moon & Apocynaceae & - & Watery latex \\
\hline 59. & Mimusops elengi L. & Saptoaceae & Mulavumaram & Milky latex \\
\hline 60. & Mirabilis jalapa L. & Nyctaginaceae & Anthimantharai & Tuber \\
\hline 61. & Mitragyna parvifolia (Roxb.) Korth. & Rubiaceae & Neerkadapai & Riparian \& Red latex \\
\hline 62. & Morinda coreiaBuch. Ham. & Rubiaceae & ManjalKadapai/Nuna & Riparian \& Gum \\
\hline 63. & Musa paradisiacal L. & Musaceae & Valai & Tuber \\
\hline 64. & Nerium olender L. & Apocynaceae & Alari & Milky latex \\
\hline
\end{tabular}




\begin{tabular}{|c|c|c|c|c|}
\hline S. No. & Botanical Name & Family & Local Name & Identification Characters \\
\hline 65. & Pavonia zeylanica (L.) Cav. & Malvaceae & Sitramutti & Root \\
\hline 66. & Pentatropsis capensis (T.f.) Bullock & Apocynaceae & Uppalankodi & Watery latex \\
\hline 67. & Pergularia daemia (Forsskal) Chiov & Apocynaceae & Uthaamanai & Milky latex \\
\hline 68. & Plecospermum spinosum (Roxb. ex Willd.) Trecul. & Moraceae & - & Milky latex \\
\hline 69. & Plumeria rubra L. & Apocynaceae & Arali & Milky latex \\
\hline 70. & Polyalthia cerasoides (Roxb.) Bedd. & Annonaceae & Senthalamaram & Red latex \\
\hline 71. & Pongamia pinnata (L.) Pierre & Fabaceae & Pungan & Riparian \\
\hline 72. & Premna tomentosaWilld. & Verbenaceae & Ponneri & Colour leaves \\
\hline 73. & Pterocarpus marsupiumRoxb. & Fabaceae & Vengai & Red latex \\
\hline 74. & Semecarpus anacardium L. & Anacardiaceae & Serra maram & Red latex, Taste \& Colour leaves \\
\hline 75. & Shorea roxburghiig. Don. & Dipterocarpaceae & Silari/Kungiliyam & Gum \\
\hline 76. & Syzgium cumini (L.) Skeels & Myrtaceae & Naval & Riparian \\
\hline 77. & Tephrosia purpurea (L.) Pers. & Fabaceae & Kozingi & Root \\
\hline 78. & Terminalia arjuna (DC.) Wight. \&Arn. & Combretaceae & Neermathi & Riparian, Resin \& leaf Glands \\
\hline 79. & Terminalia bellerica (Gaertner) Roxb. & Combretaceae & Thandrikaai & Riparian \\
\hline 80. & Terminalia crenulata Roth. & Combretaceae & Karumarudhu & Riparian \& Gum \\
\hline 81. & Terminalia tomentosa W. \& A. & Combretaceae & Pillaimarudu & Leaf Glands \\
\hline 82. & Tylophora indica (Burmit) Merr. & Apocynaceae & Kuthupalai & Milky latex \\
\hline 83. & Vitex negundo L. & Verbenaceae & Notchi & Riparian \& Colour leaves \\
\hline 84. & Withania somnifera (L.) Duanl & Solanaceae & Amakarankilangu & Root \\
\hline 85. & Wrightia tinctoria (Roxb.) R.Br. & Apocynaceae & Veppalai & Milky latex \\
\hline
\end{tabular}

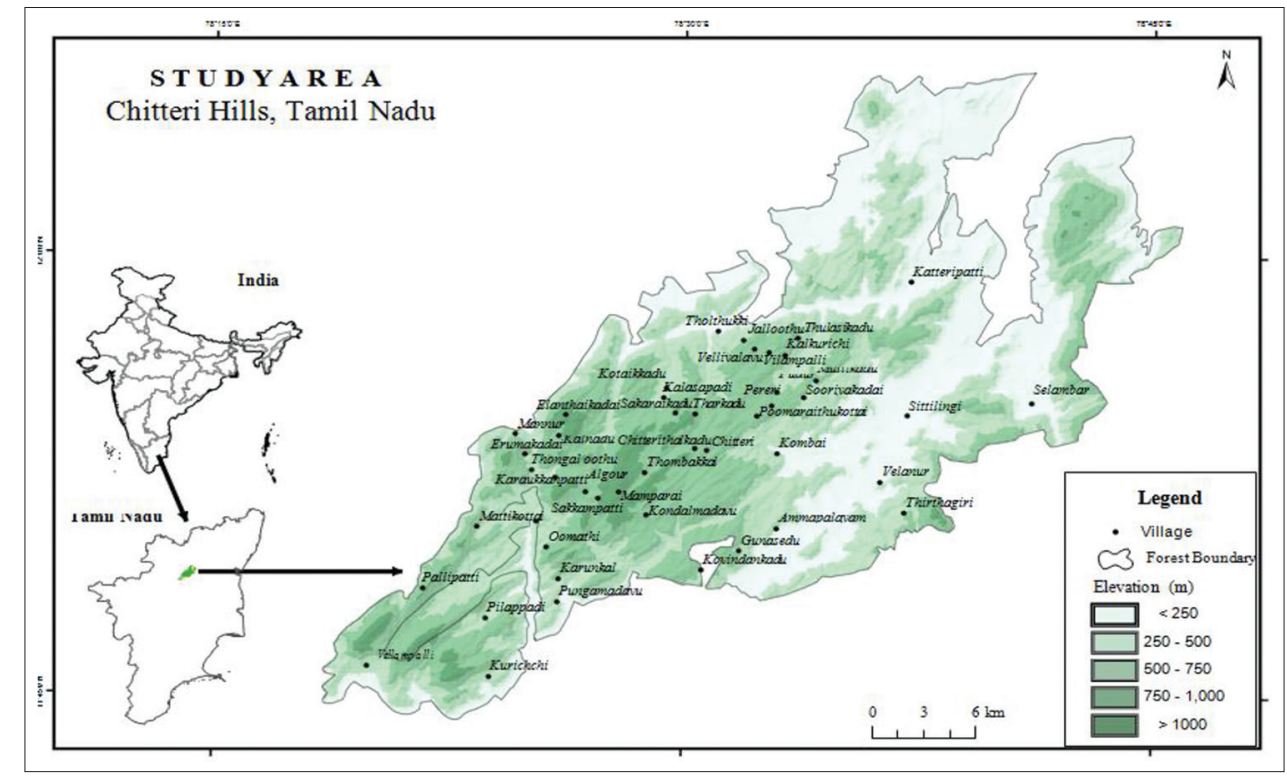

\section{Figure: 1 Study area}

the Malayali explain the periodicity of the flow of latex thus: "The rate of flow of latex from the trees is influenced by rainfall. If the species receives sufficient rainfall, the discharge of latex is copious and if the species receives insufficient rainfall, the discharge of latex is scanty". The scientific explanation for this phenomenon is as follows: "Under conditions of heavy rainfall, the cells are supersaturated with water resulting in increased turgour within the plant body. This leads to copious discharge of latex. On the other hand under conditions of drought or scanty rainfall, the cells become flaccid resulting in scanty discharge of the latex". Though they may not know the scientific basis for this phenomenon, it must be agreed that their observation is correct.

\section{Leaf characters}

Many tribes familiar with plants use sight, touch, taste, smell and sound for identification and classification of particular plant species. Tribal experience with the organoleptic properties of plants in identification comprises smell, touch and taste (Newmaster et al., 2006). Sensory perception gained by experience is an important tool for plant identification (Getchell et al., 1991; Messer, 1991).

The taste qualities that humans perceive in plants, especially bitterness, have been proposed as significant clue used in primitive societies. Malayali of Chitteri hills have clear knowledge of identification of the species in which leaf characters such as colour, taste, smell, succulence and glands form important criteria.

The Malayali of Chitteri hills also use their personal experience of taste for identification of certain species of plants. By experiencing the leaf taste they identified the following plants Andrographis paniculata, Andrographis alata, Gymnema 
sylvestre, Semecarpus anacardium, Albizia procera, Ceropegia juncea and Clerodendrum inerme. Of the aforementioned plant species, the leaves of Andrographis alata, Andrographis paniculata, Clerodendrum inerme and Semecarpus anacardium are bitter to taste.

The leaves of Gymnema sylvestre also bitter to taste. However, the leaves also have a property of rendering the taste buds neutralized so that the person who has chewed the leaves cannot taste the sweetness for quite a few hours. The tribal people gave jaggery to the present author after a bout of chewing a few leaves of Gymnema sylvestre and the experience were like having a mouthful of sand. The case of Ceropegia juncea locally called "Sempulichan" is more interesting. The taste of the stem changes, in the morning hours tastes is sour, during the noon hours, it is bitter and in the evening hours it is acidic taste.

The Malayali identify / recognize Terminalia tomentosa, Hiptage benghalensis, Mallotus philippinensis and Terminalia arjuna by the presence of glands. In fact, the vernacular name for Terminalia tomentosa is "pillai maruthu" in which the epithet 'maru' means mole. The glands often resemble 'mole'.

Colour of the leaf is another morphological criterion to distinguish between species. Malayali tribes identified eight species by leaf colour is either brown or rusty or black or copper coloured depending on the species. They are Madhuca longifolia (Red colour at new foliage), Anogeissus latifolia (Coppery colour at new foliage), Semecarpus anacardium (rusty colour below), Premna tomentosa (Yellow colour), Vitex negundo (grey pubescent colour), Litsea oleoides (brown colour), Aristolochia indica (whitish colour) and Flueggela virosa (coppery colour in tender foliage).

They identified two species by the succulence of their leaves; they are Aloe vera and Agave angustifolia. The taste and smell are criteria of medicinal and non-medicinal plants. Non-medicinal plants were more often reported to have no smell (or) taste (Classen, 1992). Taste is important in Mayan tribal medicinal plant classification, but are most likely used in combination with other markers. Taste is traditionally viewed as a mixture of four elementary qualities salty, sweet, sour and bitter. For example, plants, in the sunflower family (Asteraceae) have bitter taste because of high level of alkaloid content. This hypothesis is supported by observations of Chimpanzees using bitter Asteraceae species for treating gastro-intestinal disorders. From this observation Highland Maya tribe often treat gastrointestinal disorders with plants that are bitter (Berlin, 1992). A majority of plants are selected for medicinal use based on the property such as taste and smell. Good smelling plants are used against stomach, bitter tasting plants are applied topically for skin problems and sweet plants are given to strengthen the body and the blood (Heinrich \& Gibbons, 2001).

\section{Tuberous and Rhizomatous plants}

Malayali tribes of Chitteri hills identified underground parts of the plant species by utility. They used 19 underground plant parts belonging to 16 genera under 15 families. Roots, rhizome and tuber of these plants are used for preparing raw drug to cure ailments and food. Of these 19 species, 5 are used as food, the rest are used for medicinal properties.

Rhizomes of Dioscorea bulbifera, Dioscorea pentaphylla and Dioscorea oppositifolia are cooked and eaten. Roots of Decalepis hamiltonii are pickled and used as food adjuvant. The roots of Hemidesmus indicus yield a coolant drink called 'Nannari sharbath'.

The Malayali tribal have a wide knowledge of conserving plant species. They adopt specific strategies while harvesting plant parts for their use. For example while collecting the Dioscorea sp. they know at what stage of plant growth the rhizome is to be dugout. Based on plant and leaf growth, a Malayali knows whether the rhizome is mature or immature. They are also conscious of conserving the plant for posterity. While digging out the rhizome, they leave out some portion of rhizome with bud (called as "Moodi" in Tamil) so that it can grow in next season. This practice protects the species from extinction. They roast or boil the rhizome for consumption. The upper portion of rhizome is not used in cooking, as it cause itching sensation.

The roots of Decalepis hamiltoni is collected, washed with water, cut into small pieces and dried in the sun. The dried root pieces are pickled. Roots of Hemidesmus indicusis collected, washed with water and crushed freshly to prepare a coolant drink called 'Nannari sharbath'Malayali tribes of Chitteri hills and tribes of various regions use underground parts for their sustainable use. Tribes of Kadars, Malasars, Maduvars and Malamalaasars of Parambikulam wild life sanctuary, Kerala, listed ten edible underground parts of rhizome, tuber, corms and bulbs for cooking curry. The rhizome of Dioscorea sp. causes terrible itching sensation in ones throat if eaten raw. They peel off the outer layer, boil the rhizome in tamarind water and smear with turmeric paste to make it palatable (Yesodharan \& Sujana, 2007).

Today we know that raphides (the needle like crystals) of calcium oxalate present in the parenchyma cells of the tubers prick the tongue and mouth causing irritation. When soaked and cooked with tamarind, the tannic acid present in tamarind dissolves the crystals. It is surprising as to how the tribal people knew of the use of tamarind for this purpose.

\section{Ecological characters}

Ecological knowledge, such as where a particular plant lives is another important criterion used by the tribes for identifying plants and is perhaps limited to the geographic region. Ecology appears to play an important role in how people classified the flora and fauna of a given area (Areendran \& Rao, 2009).

Malayali tribes use landscape characters to a greater extent for identifying certain species of plants. They have accurate knowledge about species such as Ficus tomentosa, Caralluma attuneta and C. umbellata occurring in rocky terrains. Malayali tribes are knowledgeable about connecting certain species to 
the particular landscape and naming the species accordingly. The naming of plant in vernacular language is based on habitat; one typical example is Caralluma attuneta which is locally called kallumuliyan in Tamil, because this species always occurs in rocky areas.

Albizzia procera an exotic plant that occurs very rarely in Chitteri hills is another good example for this. The bark of this tree, which is used to cure all types of bone fractures, is called Koodumathurai in Tamil. The Tamil word Koodu means meeting or group and 'to join'. This species always occurs in a group of three to five. Its medicinal property joins fractured bones as well. Therefore, the Tamil vernacular name appears appropriate. Decalepis hamiltoni of Apocynaceae is another species growing in rocky areas. Malayali always look for this plant in rocky areas as their root tubes pickled as consumed as food adjuvant. The tubers have cooling properties.

To cite some more examples on their knowledge of the habitat characteristic of plants, they recognize the following plants as riparian. The field notes of the following species: Terminalia arjuna is characteristic riparian (riverbanks) (Matthew, 1995). According to Gamble it is more scarce in Carnatic region except in Tirunelveli and on the West coast; on the banks of rivers and streams. Terminalia crenulatais reported as occasional in riverbanks (Matthew, 1995), Syzygium cumini is representing variety of habitats: Shoals, riverbanks, scrub jungle (Matthew, 1995). It occur in all forest districts, both in plains and in the hills up to $6000 \mathrm{ft}$., usually along river banks and in moisture localities (Gamble \& Fisher, 1935).

Vitex negundo is common in riverbanks or fencing near households (Matthew, 1995). It is present in the dry region up to $5000 \mathrm{ft}$ in the hills, on wastelands around villages, on roadsides and the banks of streams, common (Gamble \& Fisher, 1935). Bombax ceiba occur from plains to coast, especially along riverbanks; on the deciduous belt of the hills to $800 \mathrm{~m}$ (Matthew, 1995), Drosera indica is bloom up with the monsoons (unless in perennially moist ground) (Matthew, 1995) and wet places in hills. According to Gamble Mangifera indica, occurs in ravines up to $4000 \mathrm{ft}$. Mitragyna parvifolia is often reported along rivers and foothills to $800 \mathrm{~m}$ (Matthew, 1995). Pongamia pinnata represent mostly by banks of rivers, in ravine (Matthew, 1995). It is present from coastal forest to tidal riverbanks; inland chiefly along streams and rivers in most districts in the hills up to $3000 \mathrm{ft}$ (Gamble \& Fisher, 1935) attests to their riparian nature.

Just as a field-botanist has his own scientific approach towards identifying plants in order to pick useful ones, native tribes also have their own approach based on direct observation and macro characters and this serves the purpose. Therefore, we should not dismiss the traditional knowledge of the tribal people as something without scientific basis.

In conclusion, the identification of the usefulness of a plant by organoleptic characters as practiced by the tribal people around the world may be a simple and useful tool to those who do not have a formal botanical training. This knowledge is transmitted orally from generation to generation in the tribal population. Though for scientific purposes this approach of identification of plants cannot be the sole basis, it is certainly useful as it offers supportive field characters for confirming identification. In this context, it is recommended that such indigenous knowledge is documented and incorporated in the floristic publications of the regional floras.

\section{ACKNOWLEDGEMENTS}

We thank Mr. Murugesan, Malayali tribe, Chitteri hill and Chitteri Malayali tribal village heads Tamil Nadu, India for the help rendered during the field survey for their help in identifying the plants. The authors also thank DST-PURSE (Grant No.SR/ PURSE Phase 2/16) and UGC- SAP DRS-II (No.F.5-13/2018/ DRS-II (SAP-II) for financial support.

\section{REFERENCES}

Areendran, G., \& Rao, P. (2009).Vegetation types the Southern Eastern Ghats. A Remote sensing perspective, World Wide Fund for NatureIndia.

Assogbadjo, A. E., Kyndt, T., Sinsin, B., Gheysen, G., \& Andamme, P. V. (2006). Patterns of genetic and morphometric diversity in baobab (Adansonia digitata) populations across different climatic zones of Benin (West Africa). Annals of Botany, 97, 819-830. https://doi. org/10.1093/aob/mcl043

Berlin, B. (1992). Ethnobiological classifications principles of categorization of plants and animals in traditional societies, Princeton University press, Princeton.

Classen, C. (1992). The odour of the other: Olfactory symbolism and cultural categories. Ethos, 20, 133-166. https://doi.org/10.1525/ eth.1992.20.2.02a00010

Gamble, J. S., \& Fisher, C. E. C. (1935). Flora of the Presidency of Madras, Parts 1-3 Aldard \& Son, London.

Getchell, T. V., Doty, R. C., Bartoshuk, L. M., \& Snow Jr. J. B. (eds.) (1991). Smell and taste in health disease, Raven Press, New York.

Harur Forest office Report. (2007). Tamil Nadu Forest Department, Harur range, Harur.

Heinrich, M., \& Gibbons, S. (2001). Ethnopharmacology in drug discovery: an analysis of its role and potential contribution. Journal of Pharmacy and Pharmacology, 53(4), 425-432. https://doi. org/10.1211/0022357011775712

Jain, S. K. (1995). A manual of ethnobotany (2 ${ }^{\text {nd }}$ Eds.), Jodhpur, India: Scientific Publishers.

Kakudidi, E. K. (2004). Folk plant classification by communities' crooned Kibale National park, Western Uganda. African Journal of Ecology, 22(1), 57-63. https://doi.org/10.1111/j.1365-2028.2004.00462.x

Matthew, K. M. (1995). The Flora of the Tamil Nadu Carnatic. The Rapinat Herbaium, St Joseph' College, Tiruchirapalli.

Messer, Z. (1991). Systematic and medicinal reasoning in Mitla folk botany. Journal of Ethnopharmacology, 33, 107-128. https://doi. org/10.1016/0378-8741(91)90170-I

Newmaster, S. G., Ragupathy, S., Ivanoff, R. F., \& Nirmala, C. B. (2006). Mechanisms of ethnobiological classifications. Ethnobotany, 18, 4-26.

Rao, R. R., \& Hajra, P. K. (1987). Methods of research in ethnobotany In Manual of Ethnobotany (Ed.), India: S.K. Jain Scientific publishers.

Schultes, R. E. (1962). The role of ethnobotanist in the search for new medicinal plants. Lloydia, 25, 257-266.

Yesodharan, K., \& Sujana, K. A. (2007). Wild edible plants traditionally used by the tribes in the Parambikulam wild life sanctuary, Kerala, India. Natural Product Radiance, 6(1), 73-80. 